\title{
Immunohistochemical and other prognostic factors in B cell non Hodgkin lymphoma patients, Kampala, Uganda
} Lynnette K Tumwine*1, Claudio Agostinelli2 ${ }^{2}$ Cristina Campidelli2 ${ }^{2}$ Emmanuel Othieno ${ }^{1}$, Henry Wabinga ${ }^{1}$, Simona Righi ${ }^{2}$, Brunangelo Falini ${ }^{3}$, Pier Paolo Piccaluga ${ }^{2}$, Wilson Byarugaba ${ }^{1}$ and Stefano A Pileri ${ }^{2}$

\begin{abstract}
Address: ${ }^{1}$ Department of Pathology, Makerere University, College of Health Sciences, PO Box 7072, Kampala, Uganda, ${ }^{2}$ Unit of Hematopathology, Department of Haematology and Oncological Sciences "L and A Seràgnoli"/Interdepartmental Centre for Cancer Research "G Prodi", Bologna University School of Medicine, Bologna, Italy and ${ }^{3}$ Laboratory of Haematopathology, Institute of Haematology, Perugia University School of Medicine, Perugia, Italy

Email: Lynnette K Tumwine* - tumwinelynnette@yahoo.com; Claudio Agostinelli - claudioagostinelli@gmail.com; Cristina Campidelli - cristinacampidelli@gmail.com; Emmanuel Othieno - othienoemma@yahoo.com; Henry Wabinga - hwabinga@med.mak.ac.ug; Simona Righi - pileri@aosp.bo.it; Brunangelo Falini - faliniem@unipg.it; Pier Paolo Piccaluga - pierpaolo.piccaluga@unibo.it; Wilson Byarugaba -wbyarugaba@yahoo.co.uk; Stefano A Pileri - pileri@aosp.bo.it

* Corresponding author
\end{abstract}

Published: 16 December 2009

BMC Clinical Pathology 2009, 9:1 I doi:10.1 186/1472-6890-9-1 I
Received: 23 July 2009

Accepted: 16 December 2009

This article is available from: http://www.biomedcentral.com/I472-6890/9/I I

(c) 2009 Tumwine et al; licensee BioMed Central Ltd.

This is an Open Access article distributed under the terms of the Creative Commons Attribution License (http://creativecommons.org/licenses/by/2.0), which permits unrestricted use, distribution, and reproduction in any medium, provided the original work is properly cited.

\begin{abstract}
Background: Non Hodgkin lymphomas are the most common lymphomas in Uganda. Recent studies from developed countries have shown differences in survival for the different immunophenotypes. Such studies are lacking in Africa where diagnosis is largely dependent on morphology alone. We report immunohistochemical and other prognostic factors in B cell non Hodgkin lymphoma patients in Kampala, Uganda.
\end{abstract}

Methods: Non Hodgkin lymphoma tissue blocks from the archives of the Department of Pathology, Makerere University College of Health Sciences, Kampala, Uganda, from 1991-2000, were sub typed using haematoxylin and eosin, Giemsa as well as immunohistochemistry. Using tissue micro array, I 19 biopsies were subjected to: CD3, CD5, CDI0, CD20, CD23, CD30, CD38, CD79a, CDI38, Bcl-6, Bcl-2, IRTA-I, MUMI/IRF4, Bcl-I/cyclin DI, TdT, ALKc, and Ki-67/Mibl. Case notes were retrieved for: disease stage, chemotherapy courses received and retrospective follow up was done for survival.

Results: Non Hodgkin B cell lymphomas comprised of Burkitt lymphoma [BL] (95/I I 9 ) diffuse large B cell lymphoma (I9/II9), mantle cell lymphoma (4/II9) and precursor B lymphoblastic lymphoma (I/II9).

For Burkitt lymphoma, good prognosis was associated with receiving chemotherapy, female gender and $C D 30$ positivity. Only receiving chemotherapy remained significant after Cox regression analysis. Diffuse large $B$ cell lymphomas with activated germinal centre B cell (GCB) pattern (CDI0+/-, BCL-6+/-, MUM+/ -, CDI $38+/-)$ had better survival ( 98.4 months; $95 \% \mathrm{Cl} 89.5-107.3)$ than the others $(57.3$ months; $95 \% \mathrm{Cl}$ $35.5-79.0) \mathrm{p}=0.027$ (log rank test).

Conclusions: Activated GCB diffuse large B cell lymphoma had a better prognosis than the others. For Burkitt lymphoma, not receiving chemotherapy carried a poor prognosis. Availability of chemotherapy in this resource limited setting is critical for survival of lymphoma patients. 


\section{Background}

Non Hodgkin B cell lymphomas are heterogeneous in morphology, immunophenotype and response to therapy. Recent studies have shown differences in survival based on their molecular profile[1].

In developing countries, clinically aggressive subtypes such as Burkitt and diffuse large B cell lymphoma predominate and, unfortunately, result in poor outcome[2]. Factors that influence survival in non Hodgkin lymphomas in resource poor settings include socio economic status, stage of disease at presentation and getting a full course of treatment. In Uganda, several studies have described clinical factors associated with outcome of Burkitt lymphoma $[3,4]$.

In the developed countries, several methods including gene profiling and immunohistochemistry have been used for predicting prognosis $[5,6]$. Using the gene expression profile of germinal centre B and activated B cell, diffuse large B cell lymphoma (DLBCL) was subdivided into 3 prognostic groups. However, there are several draw backs of gene expression profiling especially in resource constrained countries such as Uganda. It requires the use of optimally cryopreserved or fresh tissues as well as DNA micro array technology which is more costly than immunohistochemistry on paraffin sections.

Recently, several workers have used germinal centre and activated B cell immunohistochemical markers on paraffin embedded tissue blocks to classify DLBCL into three prognostic groups $[7,8]$.

These include: (a) activated non GCB (CD10-, Bcl-6-, MUM1/IRF4, \pm CD138+); (b)activated GCB (CD10+, Bcl6+, MUM1/IRF4, \pm CD138+); and (c) non activated GCB (CD10+, BCl-6+, MUM1/IRF4-, CD138-). They showed that patients with a germinal centre $\mathrm{B}$ cell profile have a much better prognosis than those with the activated B cell type. Such studies have hitherto not been carried out in Uganda. We report immunohistochemical and other prognostic factors in B cell non Hodgkin lymphoma patients in Kampala, Uganda

\section{Methods}

\section{Study design and sampling}

A cross sectional descriptive design was used for lymphoma diagnosis and immunophenotyping, while a retrospective cohort was used to determine survival. For the cross sectional study, haematoxylin and eosin and Giemsa staining was carried out in the Department of Pathology, Makerere University and immunohistochemistry in the Unit of Hematopathology, Institute of Hematology and Clinical Oncology "L. \& A. Seràgnoli", Bologna University School of Medicine, Bologna, Italy. One hundred and twenty nine patients' biopsies diagnosed between 19912000 as non Hodgkin lymphoma were sub typed using tissue microarray (TMA) and immunohistochemistry with CD3, CD5, CD10, CD20, CD23, CD30, CD38, CD79a, CD138，Bcl-6，Bcl-2，IRTA-1, MUM1/IRF4，Bcl-1/cyclin D1, TdT, ALKc, and Ki-67/Mib1.

For the retrospective cohort study we retrieved patients' case notes from the Uganda Cancer Institute in order to obtain details of the patients' disease stage, type of chemotherapy, number of courses received, whether dead or alive, time to death. Cancer registry data was also used when the addresses of the patients fell within Kyadondo County, the area covered by the Kampala Cancer Registry. One of us (LKT) and two research assistants followed up patients whose survival status was not clear. The follow up involved tracing patients to their homes (district, subcounty, parish and village) in the different regions of Uganda.

The patients had been treated at the Uganda Cancer Institute which is the oldest unit for cancer treatment in the country. It began as a centre for the treatment of Burkitt lymphoma patients in the 1960s and has two units: the solid tumor treatment unit and the lymphoma treatment centre. Those with Burkitt lymphoma received COM[9] (cyclophosphamide, vincristine, intrathecal methotrexate) whereas those with other non Hodgkin lymphomas received CHOP (cyclophosphamide, adriamycin, vincristine and prednisolone).

\section{Tissue micro array construction (TMA)}

Haematoxylin and eosin (H\&E) stained slides were used to identify the representative tumor fields that were marked and correspondingly identified on the tissue blocks.

Tissue cylinders of diameter of $1 \mathrm{~mm}$ were punched from the marked areas on each block and incorporated into a recipient paraffin block using a precision instrument, the tissue arrayer (Beecher Instruments, Silver Spring, Maryland, USA). For adequate sampling each specimen was represented in duplicate using $1 \mathrm{~mm}$ cores in the recipient block. Three TMA recipient blocks were made; two of these had 48 punches each of Burkitt lymphoma while the other one had 33 punches of other non Hodgkin lymphomas.

\section{Immunohistochemistry}

Four- $\mu \mathrm{m}$ thick sections were cut from TMAs, coated on electrically charged slides, re-hydrated, and submitted to antigen retrieval in ethylene diamine tetra acetic acid (EDTA) $1 \mathrm{mM}$ (pH 8.0) by micro-waving twice for $5 \mathrm{~min}$ utes at either 750 or $900 \mathrm{~W}$, that was very efficient according to previous experience[10]. 
After cooling, the slides were put on a TechMate 500 immunostainer and incubated for 30 minutes at room temperature with antibodies against CD3, CD5, CD10, CD20, CD23, CD30, CD38, CD79a, CD138, Bcl-6, Bcl-2, IRTA-1, MUM1/IRF4, Bcl-1/cyclin D1, TdT, ALKc, and Ki67/Mib1. Details on the antibodies, sources, dilutions and antigen retrieval are listed in Table 1.

The antibodies were detected by either the alkaline phosphatase anti-alkaline phosphatase immunocomplexes (APAAP) technique or the Envision+ technique [11].

\section{Data management, analysis and statistical issues}

For the descriptive cross sectional study, a sample size of 112 was calculated using a formula by Kish and Leslie[12]. In this calculation we assumed that the prevalence of B cell non Hodgkin's lymphoma in the total population of non-Hodgkin lymphoma was $92.1 \%$ according to a Ugandan study[2] with a precision of $5 \%$ and $95 \%$ confidence interval.

For the retrospective cohort design, a sample size of 52 was calculated using a formula by Fleiss with $80 \%$ power and $95 \%$ confidence interval [12]. We assumed the expected outcome among patients with NHL of germinal centre origin would be $86 \%$ and the expected outcome among patients with NHL of non germinal centre origin would be $63 \%$.

Data was collected and entered into the computer using EPI INFO software (supplied by CDC and WHO) for storage and initial analysis. Further analysis was done using

Table I: Primary antibodies used for the study.

\begin{tabular}{|c|c|c|c|c|}
\hline Antibody & Clone & Source & Antigen retrieval & Dilution \\
\hline CD3 & SP7 & Immunotech & EDTA $750 \mathrm{~W}$ & $\mathrm{I}: 250$ \\
\hline CD5 & $54 / F 6$ & Dako & EDTA $900 \mathrm{~W}$ & $\mathrm{I}: 10$ \\
\hline CDIO & $56 \mathrm{C} 6$ & Novocastra & EDTA $900 \mathrm{~W}$ & $\mathrm{I}: 5$ \\
\hline CD20 & $\mathrm{L} 26$ & Dako & EDTA $750 \mathrm{~W}$ & $\mathrm{I}: 200$ \\
\hline $\mathrm{CD} 23$ & $|B| 2$ & Novocastra & EDTA $900 \mathrm{~W}$ & $\mathrm{I}: 30$ \\
\hline CD30 & Ber- $\mathrm{H}_{2}$ & Prof. Falini * & EDTA $900 \mathrm{~W}$ & $1: 3$ \\
\hline $\mathrm{CD} 38$ & SPC 32 & Novocastra & EDTA $750 \mathrm{~W}$ & $\mathrm{I}: 10$ \\
\hline CD79a & $J C B \mid I 7$ & Prof. Mason $\S$ & EDTA $750 \mathrm{~W}$ & $1: 10$ \\
\hline CDI38 & - & Neomarkers & EDTA $900 \mathrm{~W}$ & $\mathrm{I}: 20$ \\
\hline BCL-I & SP4 & Neomarkers & EDTA $900 \mathrm{~W}$ & $\mathrm{I}: 20$ \\
\hline$B C L-6$ & PG-B6P & Prof. Falini * & EDTA $900 \mathrm{~W}$ & Undiluted \\
\hline BCL-2 & 124 & Prof. Mason§ & EDTA $900 \mathrm{~W}$ & $1: 3$ \\
\hline IRTA-I & Mum2EC & Prof. Falini * & EDTA $900 \mathrm{~W}$ & $1: 2$ \\
\hline MUMI/IRF4 & - & Prof. Falini * & EDTA $900 \mathrm{~W}$ & $1: 2$ \\
\hline TdT & - & Dako & None & $\mathrm{I}: 30$ \\
\hline ALKc & - & Prof. Falini * & EDTA $900 \mathrm{~W}$ & $1: 2$ \\
\hline $\mathrm{Ki}-67$ & Mib-I & Dako & EDTA $900 \mathrm{~W}$ & $\mathrm{I}: 20$ \\
\hline
\end{tabular}

* Kindly provided by Prof. Brunangelo Falini, Perugia University, Perugia, Italy.

$\S$ Kindly provided by Prof. David Y. Mason, Oxford University, Oxford, before his premature death. 
SPSS software. For continuous variables, the relevant measures of central tendency (means for normally distributed data and medians and inter-quartile ranges for skewed data) were used to explore the data.

Kaplan-Meier curves and the log rank test were used to determine survival. To determine factors associated with overall survival, univariate and multivariate Cox hazards regression analysis was carried out. A p value of less than 0.05 was considered significant.

\section{Ethical issues}

Permission to carry out the study was obtained from the Makerere University Faculty of Medicine Research and Ethics Committee.

\section{Study limitations}

This was a retrospective rather than prospective study making it difficult to get good socio-demographic information. Clinical outcome predictors such as the International prognostic Index were not complete. Lactate dehydrogenase was not routinely done in the patients.

\section{Results}

\section{Background characteristics}

The median age was 9.0 (inter-quartile range 6- 15.5) years. The youngest patient was 2 years and the oldest was 64 years. The mean age was 15.7 (SD 15.5) years.

As expected, $79.8 \%$ of the patients had Burkitt lymphoma, followed by diffuse large B cell lymphoma (16.0\%), mantle cell lymphoma (3.4\%), and precursor B lymphoblastic lymphoma (0.8\%) (Table 2)

\section{Burkitt lymphoma survival}

The male patients had a mean survival of 3.3 (95\% CI 0.81-5.8) months while the females had a mean survival of 17.7 (95\% CI 8.3-27.0) months, $\mathrm{p}=0.028$. The CD30 negative patients had a mean survival of 6.8 (95\% CI 0.0 - 14.7) while the CD30 positive had a mean survival of 12.3 (95\% CI 7.3 - 17.3), $\mathrm{p}=0.0169$.

Only receiving chemotherapy remained significant after Cox regression analysis (Table 3 )

\section{Burkitt lymphoma survival by EBER status}

Overall 97.3\% were EBER positive. Survival information was available on 25 patients whose EBER results were available. Of these, 24 (96\%) were EBER positive. The mean survival for the 24 was $9.28(95 \%$ CI 3.27-15.29 months). However there was only one EBER negative patient whose survival was 1.2 months.

\section{Burkitt lymphoma: EBER status and CD30 positivity}

A total of 55 cases had both CD30 and EBER results. Of the 24 CD30 positive, $23(95.8 \%)$ were EBER positive. Of the 31 CD30 negative, 30(96.8\%) were EBER positive. The difference was not statistically significant $(\mathrm{p}=0.83)$; and because of this, the EBER result was not entered into Cox regression analysis.

\section{Diffuse large B cell lymphoma survival}

Diffuse large $B$ cell lymphomas with activated germinal centre B cell (GCB) pattern (CD10+/-, BCL-6+/-, MUM+/, CD138+/-) had better survival (98.4 months; 95\% CI 89.5 - 107.3) than the others (57.3 months; 95\% CI 35.5 79.0) $\mathrm{p}=0.027$ (log rank test) (Figure 1)

Table 2: Distribution of B cell non Hodgkin lymphoma by diagnosis and immunophenotype

\begin{tabular}{lllll}
\hline $\begin{array}{l}\text { Diagnosis } \\
\text { Marker }\end{array}$ & $\begin{array}{l}\text { Burkitt } \\
\text { lymphoma } \\
\text { (\%positive) }\end{array}$ & $\begin{array}{l}\text { Diffuse large B cell lymphoma } \\
\text { (\% positive) }\end{array}$ & $\begin{array}{l}\text { Precursor B lymphoblastic } \\
\text { lymphoma }\end{array}$ & Mantle cell lymphoma \\
\hline CD10 & $74 / 79(93.7)$ & $3 / 18(16.7)$ & $1 / 1$ & $0 / 4$ \\
CD20 & $95 / 95(100)$ & $19 / 19(100)$ & $1 / 1$ & $4 / 4$ \\
BCL6 & $46 / 69(66.7)$ & $2 / 19(10.5)$ & $0 / 1$ & $1 / 4$ \\
CD79a & $48 / 68(70.6)$ & $6 / 16(37.5)$ & $1 / 1$ & $2 / 4$ \\
CD38 & $35 / 68(51.5)$ & $3 / 16(18.8)$ & $0 / 1$ & $0 / 4$ \\
MUM-I/IRF4 & $1 / 77(1.3)$ & $4 / 18(22.2)$ & $0 / 1$ & $0 / 4$ \\
EBER & $79 / 86(91.9)$ & $6 / 17(35.3)$ & $1 / 1$ & $0 / 4$ \\
Tdt & $0 / 95(0)$ & $0 / 19(0)$ & $1 / 1$ & $0 / 4$ \\
BCL2 & $6 / 79(7.6)$ & $7 / 19(36.8)$ & $1 / 1$ & $4 / 4$ \\
CD3 & $0 / 86(0)$ & $0 / 19(0)$ & $0 / 1$ & $0 / 3$ \\
CD138 & $43 / 95(45.3)$ & $10 / 15(66.7)$ & $0 / 1$ & $4 / 4$ \\
CD30 & $35 / 95(36.8)$ & $2 / 17(11.8)$ & $0 / 1$ & $0 / 4$
\end{tabular}


Table 3: Results of Cox regression analysis for survival, Burkitt lymphoma cases, Uganda

\begin{tabular}{lll}
\hline Variable & Hazard's ratio $(95.0 \% \mathrm{CI})$ & p value \\
\hline Received chemotherapy & $0.15(0.001-0.195)$ & 0.001 \\
\hline CD30 & $0.427(0.098-1.863)$ & 0.257 \\
\hline Male & $4.207(0.775-22.84)$ & 0.096 \\
\hline
\end{tabular}

$\mathrm{Cl}$ Confidence Intervals

\section{Discussion}

The aim of this paper was to classify B cell non Hodgkin lymphomas in Uganda using immunohistochemical markers and correlate this and other factors to patient outcome.

In the current study, B cell non Hodgkin lymphomas seem to be affecting mainly young people, with a median age of 9 years. The mean age was 15.7 years consistent with results of previous similar studies from Uganda [2], but much lower than that reported from Kenya by Cool and Bitter [13].

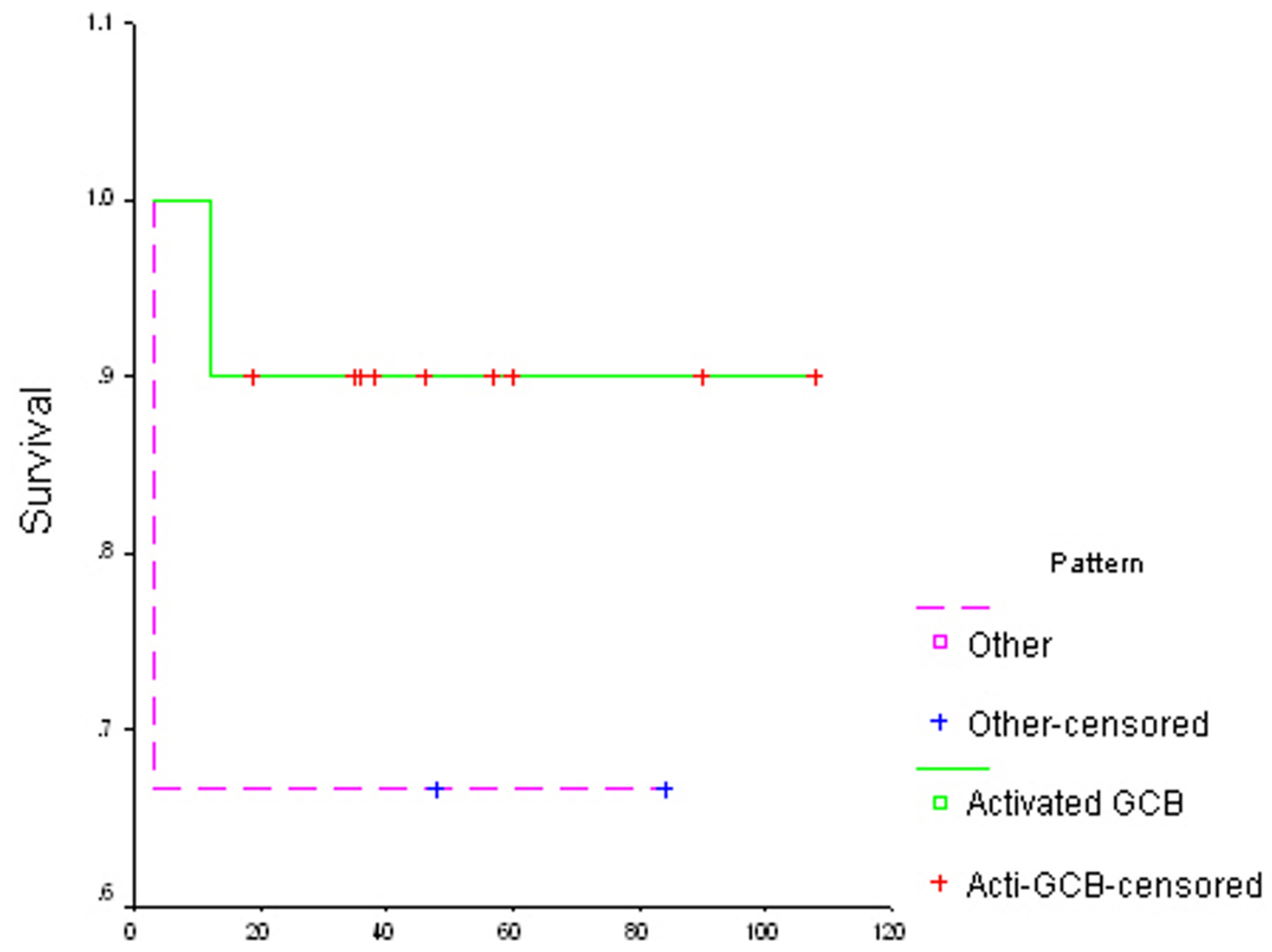

Time sunvived in months

Figure I

Kaplan Meier survival curve for patients with Diffuse Large B Cell Lymphoma, Kampala, Uganda. 


\section{Overall survival of patients with B cell non Hodgkin lymphomas \\ Burkitt lymphoma}

The overall survival of patients with B cell non Hodgkin lymphomas was 60 months but only 12.7 months in patients with Burkitt lymphoma. This survival of patients with Burkitt lymphoma in this series is surprisingly much lower than what was reported from the same centre in the 1970 s and 1980s by Olweny and others [14]. This difference could possibly be due to the fact that Olweny's studies were done on a cohort that was meticulously followed up, unlike the current investigation in which we studied routine patients who came to the Uganda Cancer Institute for treatment and who were not particularly followed up or sought after to complete treatment [14].

In the current study factors associated with better survival included receiving chemotherapy, female gender and CD30 positivity.

As expected those who did not receive chemotherapy died within the first month after presentation to hospital. The possible reason for this is that the Uganda Cancer institute is grossly under funded and therefore patients have to buy anticancer drugs from private pharmacies. Unfortunately, most patients are poor and therefore cannot afford the drugs[15]. Many patients are referred from rural areas to the main cancer treatment centre in Kampala at the Uganda Cancer Institute and this constitutes further delay.

In Uganda, the standard regimen for treating Burkitt lymphoma is cyclophosphamide, vincristine, prednisolone (COM) and intrathecal methotrexate for central nervous system disease prophylaxis $[9,14]$. In our study, those who received chemotherapy with COM had a better overall survival than those who did not. Endemic Burkitt lymphoma is characterised by a very high proliferative index nearing $100 \%$, and the disease is very rapidly progressive with a doubling time of 24 hours and is fatal if not treated early with intensive chemotherapy regimens [16].

Another factor associated with poor survival was male gender. Similar observations have been made by other authors in studies on childhood cancers $[17,18]$. The reasons for the difference in prognosis between males and females among patients with Burkitt lymphoma are not very clear. However, one explanation is that males have an inherent tendency to have higher rates of cell division than females [19]. The fact that the growth rate of the male embryo is higher than that of the female [20] has been suggested as lending credence to this hypothesis [21].

Of interest in our study was the high CD30 positivity $(37 \%)$ in Burkitt lymphoma.
This is different from results of a previous study in the United Kingdom where CD30 positivity in Burkitt lymphoma patients was not so high: Jones and others found $18 \%$ (3/17) CD30 positivity in childhood Burkitt lymphoma in the West Midlands, UK [22,23].

In our study CD30 positive patients had a better survival than those who were CD30 negative. Previously, CD30 positivity has not been associated with good prognosis in Burkitt lymphoma [24]. Several authors have suggested that the CD30-CD30L (ligand) interaction may have a role in some non Hodgkin lymphomas including Burkitt lymphoma [23,25]. Kanavaros et al 1992 found that many CD30 positive non Hodgkin lymphomas were EBV positive [25].

In our patients, CD30 positivity was found among those with both "typical morphology and plasmacytoid features." [2]. As we noted previously, this might be related to the "postulated complex pathogenesis of BL" in Africa [26]. Of interest is the multistep oncogenetic mechanism (proposed by Klein [27]) "in which there is de-regulation of MYC gene and subsequent development of a malignant clone." However, it appears that more studies are needed to shed more light on the specific role of CD30 in Burkitt lymphomas.

\section{Survival of patients with DLBCL}

In our study, diffuse large B cell lymphomas fell into three distinct groups with independent prognostic significance. These included: (a) non activated GCB, (b) activated GCB, and (c) activated non GCB. These are similar to the groups identified by researchers in the developed countries [7].

Whereas studies in the developed countries have found that non activated GCB had the best prognosis [7], in our study we have found that patients with activated germinal centre B- cell lymphoma had the best prognosis. The reasons for this difference are not clear and given the small numbers will have to be confirmed by larger studies. The difference could also be a reflection of yet unrecognized molecular heterogeneity in the tumors [28].

The overall survival of the patients with DLBCL in our study was lower than that reported from the developed countries. This could be related to the fact that in Uganda, patients with DLBCL are treated with cyclophosphamide, adriamycin, vincristine, prednisolone (CHOP) rather than the more effective cyclophosphamide, adriamycin, vincristine, prednisolone plus Rituximab (CHOP-R) that has become the standard of care in the developed countries [29]. 


\section{Conclusion}

Immunohistochemistry on paraffin embedded tissue blocks using selected GCB and activation markers has yielded important information that predicts the outcome of patients with non Hodgkin B cell lymphomas in Uganda. Generally the Ugandan patients studied had a very poor prognosis. A number of factors including lack of timely chemotherapy seem to be responsible for this.

\section{Conflict of interests}

The authors declare that they have no competing interests.

\section{Authors' contributions}

LKT conceived the idea, collected the data, and wrote the manuscript. CA collected data CC collected data. EO contributed tissue blocks and collected data. HW contributed tissue blocks and survival data. SR did the immunohistochemistry and in situ hybridisation. WB reviewed and revised the manuscript. PPP reviewed and revised the manuscript. SAP reviewed and revised the manuscript. All authors read and approved the final version of the manuscript.

\section{Acknowledgements}

The authors are grateful to the research assistants, Ms. Covia Atuheire and Mr. William Barugahare who retrieved the survival data through retrospective follow up in the villages. Special thanks go to Bologna University, Unit of Haematopathology, "L" and "A" Seragnoli who provided immunohistochemistry. This work was supported by BolognAIL (Bologna, Italy), PRINCofin (Rome, Italy), AIRC (Milan, Italy), Fondazione Cassa di Risparmio in Bologna (Bologna, Italy) and the Carnegie Corporation of New York.

\section{References}

I. Camilleri-Broet S, et al.: A uniform activated B-cell like immunophenotype might explain the poor prognosis of primary central nervous system lymphomas:analysis of 83 cases. Blood 2006, 107:190-196.

2. Tumwine L, et al.: B-cell non-Hodgkin Iymphomas in Uganda: an immunohistochemical appraisal on tissue microarray. Hum Pathol 2008, 39(6):817-823.

3. Rosenwald $A$, et al.: The use of molecular profiling to predict survival after chemotherapy for diffuse large B cell lymphoma. N Eng J Med 2002, 346(25): 1937-1947.

4. Rimsza L, et al.: Gene expression predicts overall survival in paraffin embedded tissues of diffuse large B cell lymphoma. Blood 2008, I I 2(8):3425-3433.

5. Dave $S$, et al:: Molecular Diagnosis of Burkitt's lymphoma. $N$ Eng J Med 2006, 354(23):2431-2442.

6. Hummel $M$, et al: A biologic definition of Burkitt's lymphoma from transcriptional and genomic profiling. N Eng J Med 2006, 354(23):2419-2430.

7. Chang C, et al.: Immunohistochemical expression patterns of germinal center and activation B-cell markers correlate with prognosis in diffuse large B-cell lymphoma. Am J Surg Pathol 2004, 28(4):464-470.

8. Zinzani $P$, et al.: Identification of outcome predictors in diffuse large B-cell lymphoma. Immunohistochemical profiling of homogeneously treated de novo tumors with nodal presentation on tissue microarrays. Haematologica 2004, 90(3):34I-347.

9. Orem J, et al.: Clinical characteristics and outcome of children with Burkitt lymphoma in Uganda according to HIV infection. Pediatr Blood Cancer 2009, 52(4):455-458.
10. Pileri S, Roncador G, Ceccarelli Cea: Antigen retrieval techniques in immunohistochemistry: comparison and different methods. J Pathol 1997, I83:1 16-123.

II. Sabattini E, Bisgaard K, Ascani S: Envision Plus: A new immunohistochemical method of choice for diagnostics and research. Critical comparison with the APAAP, ChemMate, CSA, LABC and SABC techniques. I Clin Pathol 1998, 5 I:506-5 II.

12. Kirkwood B: Calculation of required sample size. London: Blackwells Science Limited; 1988.

13. Cool C, Bitter ML: The malignant lymphomas of Kenya. Hum Pathol 1997, 28(9): I026-1033.

14. Olweny $C$, et al.: Long term experience with Burkitt's lymphoma in Uganda. Int J Cancer I980, 26(3):26I-6.

15. Orem J, Casper C, Sessle E: The Uganda program on cancer and infectious diseases (UPCID). Creating a centre of excellence for cancer research, training and care in Africa Seattle: The Fred Hutchinson Cancer Research Centre; 2008:6.

16. Lukande $R$, Wabinga $H$, Tumwine L: Burkitt's lymphoma in Uganda: the role of immunohistochemistry in diagnosis. East Afr Med J 2008, 85(5):207-2I 2.

17. Pui C, et al:: Sex differences in prognosis for children with acute lymphoblastic leukaemia. I Clin Oncol 1999, I 7(3):818-824.

18. Hasselblom $S$, et al:: The impact of gender, age and patient selection on prognosis and outcome in diffuse large B-cell lymphoma- a population- based study. Leuk Lymphoma 2007, 48(4):736-745.

19. Preston-Martin $S$, et al: Increased cell division as a cause of human cancer. Cancer Res 1990, 50(23):74I5-742I.

20. Mittwoch U: Blastocysts prepare for the race to be male. Human Reproduction 1993, 8( I 0): I550-I555.

21. Dorak T: Sex differential in childhood cancer. Gender effects in Cancer 2008.

22. Jones $\mathrm{T}$, et al.: Immunophenotypic analysis of childhood Burkitt's lymphoma in the West Midlands I957-1986. J Clin Pathol 1995, 48:2225.

23. Gruss $\mathrm{H}$, et al.: Pleiotropic effects of the CD30 ligand on CD30expressing cells and lymphoma cell lines. Blood 1994, 83(8):2045-2056.

24. Horie R, Watanabe T: CD30: expression and function in health and disease. Seminars in Immunology 1998, I 0(6):457-470.

25. Kanavaros $P$, et al.: High incidence of EBV genome in CD30positive non- Hodgkin's lymphomas. the Journal of Pathology 1992, I 68(3):307-315.

26. Bosch $C$ Van den: Is endemic Burkitt's lymphoma an alliance between three infections and a tumour promoter? The Lancet Oncology 2004, 5:738-746.

27. Klein G: Lymphoma development in mice and humans: diversity of initiation is followed by convergent cytogenetic evolution. Proc Natl Acad Sci USA 1979, 76:2442-2446.

28. Alizadeh A, et al.: Distinct types of diffuse large B-cell lymphoma identified by gene expression profiling. Nature 2000 , 403(6769):503-5I I.

29. Halaas J, et al.: R-CHOP-I4 in patients with diffuse large B-cell lymphoma: feasibilty and preliminary efficacy. Leuk Lymphoma 2005, 46(4):54I-547.

\section{Pre-publication history}

The pre-publication history for this paper can be accessed here:

http://www.biomedcentral.com/1472-6890/9/11/prepub 